\title{
Bases psicopatológicas do crime violento - estudo caso-controle retrospectivo de pacientes delirantes criminosos e não-criminosos
}

\author{
Psychological bases of violent crime - a retrospectiv case-control \\ study of criminal and non-criminal delusional patients \\ Eduardo Henrique Teixeira', Paulo Dalgalarrondo?
}

\section{RESUMO}

Objetivo: Estudar os aspectos do delírio que podem estar relacionados à ocorrência de crime violento por pacientes delirantes. Métodos: Estudo caso-controle retrospectivo comparando dois grupos de 30 pacientes psicóticos delirantes. O grupo estudado consiste de pacientes delirantes internados em uma Casa de Custódia do estado de São Paulo, Brasil, e o grupo comparado consiste de pacientes de enfermarias psiquiátricas comuns. Foram utilizadas as escalas PANSS, MINI e MMDAS. Resultados: Em relação às dimensões do delírio, o grupo-caso teve menor pontuação em "inibição de ação por causa do delírio" e "afeto negativo". Conclusão: Delírios que induzem a inibição de ações aparentemente também reduzem o potencial de ações violentas e, ao contrário do que se afirma correntemente, pacientes

\section{Palavras-chave}

Transtornos psicóticos, delírio, crime.

\section{Keywords}

Psychotic disorder, delusion, crime. delirantes assustados ou com outros afetos negativos associados ao delírio parecem cometer menos atos violentos. Portanto, fatores intrínsecos inerentes a algumas dimensões do delírio podem ser relevantes na ocorrência de crimes violentos cometidos por pacientes psicóticos.

\begin{abstract}
Objective: To study aspects of the delusion that can be related to the occurrence of violent crime for delusion patients. Methods: A retrospective case-control study comparing two groups of 30 psychotic delusional patients. The study group consisted of delusional patients imprisoned in a high security forensic hospital in the state of São Paulo, Brazil, and the patients in the comparative group were enrolled in common psychiatric wards. The PANSS, the MINI and the MMDAS scales were used. Results: Regarding the dimensions of delusions, the study group had lower scores in "refraining from acting because of belief" and "negative affect". Conclusion: Delusions that induce inhibition of actions apparently also reduce the potential for violent acts and, contrary to current beliefs, delusional patients who are frightened or who have other negative affects associated with delusional ideas appear to commit fewer violent acts. Therefore, intrinsic factors inherent to some delusion dimensions may be relevant in the occurrence of violent crimes committed by psychotic patients.
\end{abstract}

1 Departamento de Psicologia Médica e Psiquiatria da Faculdade de Ciências Médicas da Universidade Estadual de Campinas (FCM-UNICAMP). 
A relação entre transtorno mental grave e violência é muito complexa e, não obstante os avanços na metodologia utilizada em pesquisa psiquiátrica, o assunto continua a gerar vários debates ${ }^{1-3}$, freqüentemente porque existe um intervalo de tempo considerável entre o crime e a avaliação adequada dos sujeitos que os cometeram, incluindo seu estado mental, diagnóstico e circunstâncias ambientais.

Por causa de um pequeno subgrupo, é aceito, atualmente, que alguns indivíduos com esquizofrenia sejam mais prováveis de serem violentos do que a população geral. No entanto, a porcentagem de toda a violência social atribuída à psicose e à esquizofrenia é pequena, geralmente abaixo de $10 \% \%^{2,4}$.

Um ato grave de violência, definido como agressão ou ataque que causem dano físico sério (critério para violência grave de MacArthur), cometido por uma pessoa com transtorno mental grave, é evento relativamente raro ${ }^{5}$. Além disso, a literatura sobre os transtornos mentais graves e a violência apresenta dificuldades metodológicas, em virtude das avaliações diagnósticas imprecisas, da presença de comorbidades (particular uso/abuso de substâncias psicoativas) e das variações culturais na percepção e na construção do que seja de fato ato criminoso, assim como a falta de precisão na definição da própria violência ${ }^{6,7}$.

Estudos realizados em diferentes países (Estados Unidos, Inglaterra, Austrália e República Checa) com prisioneiros que tinham cometido crimes violentos indicaram prevalência mais elevada da esquizofrenia neste grupo (prisioneiros) em comparação com a população geral, não obstante as limitações das generalizações por causa dos vieses de tais estudos ${ }^{8-15}$. Outros estudos, entretanto, indicaram associação negativa entre psicose e violência. Stuart e Arboleda-Florez ${ }^{16}$ reanalisaram dados de um estudo realizado no Canadá, em 1992, no qual a prevalência dos transtornos mentais entre prisioneiros foi avaliada e somente pequena proporção de comportamento criminoso violento pôde ser atribuída aos pacientes psiquiátricos. Na Inglaterra, Taylor et al. ${ }^{17}$ estudaram as populações de pacientes de três hospitais psiquiátricos "especiais", caracterizados por ser de segurança elevada e de oferecer tratamento aos pacientes considerados de elevado risco de violência. Os registros de 1.750 pacientes foram examinados e foi encontrada maioria (53\%) de esquizofrenia e transtorno delirante. Observaram que os principais fatores motivadores para o crime foram os sintomas delirantes, os quais conduziam mais freqüentemente a atos violentos do que a atos triviais. As alucinações não tiveram o mesmo efeito quando a atividade delirante não estava presente.

Link e Stueve ${ }^{18}$ compararam a presença e as conseqüências de sintomas psicóticos de uma amostra psiquiátrica e da comunidade. Concluíram que delírios com conteúdo de controle ou paranóide - chamados de sintomas de ameaça/controle e domínio (TCO) - estavam associados fortemente com os atos de violência. Resultados semelhantes foram, também, relatados por Cheung et al. ${ }^{19}$, que estudaram 31 pacientes esquizofrênicos definidos como violentos e comparados a 31 pacientes não-violentos com o mesmo diagnóstico. Em ambos os grupos, a comorbidade para abuso de substância psicoativa não estava presente. A fenomenologia das alucinações auditivas foi cuidadosamente avaliada utilizando o MUPS (Escala de Percepções Incomuns do Instituto de Pesquisa de Saúde Mental) e os delírios utilizando o MADS (Escala de Avaliação de Delírios de Maudsley). Os pacientes do grupo violento foram mais afetados por delírios persecutórios do que aqueles do grupo não-violento, enquanto pacientes do grupo não-violento foram mais afetados por delírios de grandeza do que aqueles do grupo violento.

Appelbaum, Robbins e Roth ${ }^{20}$ examinaram pacientes delirantes com história evidente de violência e encontraram alto nível de convicção em delírios com conteúdo de grandeza e religiosidade. No entanto, em outro trabalho para confirmar os resultados precedentes que relacionavam o tipo e o conteúdo do delírio com crime violento, Appelbaum, Robbins e Monahan ${ }^{21}$ realizaram um estudo prospectivo e encontraram resultados negativos. Este estudo acompanhou 1.136 pacientes por um ano após a alta hospitalar. Utilizando o MMDAS (Escala de Avaliação de Delírios de MacArthur-Maudsley), nenhuma diferença foi encontrada na taxa de violência entre pacientes delirantes e não-delirantes.

Para determinar possíveis associações entre esquizofrenia, delírio e crime, muitos estudos utilizaram registros médicos ou documentos da polícia, geralmente retrospectivos e muito tempo após a ocorrência do ato violento. Estas dificuldades são ainda mais sérias quando são considerados os crimes violentos que ocorrem durante o período prodrômico de um estado de esquizofrenia ${ }^{22}$.

Apesar de tais limitações metodológicas, alguns aspectos do estado mental agudo parecem estar relacionados à presença de comportamento violento entre pacientes psicóticos. Enquanto alguns estudos mostraram que os delírios com conteúdo de ameaça e controle podem estar relacionados com desencadeantes do comportamento violento, foi sugerido que aspectos inerentes às diferentes dimensões dos delírios pudessem estar envolvidos em alguns atos criminosos ${ }^{18,21,23,24}$.

Conseqüentemente, o presente estudo foi realizado para avaliar as relações possíveis entre o tipo e as dimensões dos delírios e do crime violento. As hipóteses principais são a de que conteúdo e certas dimensões do delírio (convicção, afeto negativo, atuação no delírio, inibição de ação por causa do delírio, preocupação e penetração) estavam mais associados a crime violento. 


\section{MÉTODOS}

Este é um estudo caso-controle retrospectivo e comparativo, no qual dois grupos de 30 homens delirantes foram analisados. O grupo estudado consistiu de pacientes delirantes de uma Casa de Custódia do estado de São Paulo, e os pacientes do grupo comparado foram selecionados de enfermarias psiquiátricas comuns.

O grupo estudado consistiu de pacientes selecionados em decorrência de terem cometido crime violento relacionado à atividade delirante e tiveram diagnóstico do espectro da esquizofrenia. O grupo comparado foi composto por pacientes com transtornos do espectro da esquizofrenia, com clara atividade delirante e que nunca havia cometido crime violento. Os sujeitos foram selecionados com base em sua história de crimes violentos, na ocorrência de delírio no passado e no presente e no diagnóstico de transtorno do espectro da esquizofrenia. Todos os pacientes foram entrevistados pelo psiquiatra (EHT) na instituição onde eles foram selecionados.

A fim de identificar a atividade delirante nesses grupos, os pacientes tiveram seus sintomas do passado e do presente confirmados no início da entrevista. O critério de inclusão exigia que todos os pacientes ainda estivessem delirantes, mesmo sob uso de medicação. Um critério adicional de inclusão para o grupo estudado era a exigência de que os pacientes deveriam manter atualmente o mesmo conteúdo de delírio do período do crime. A confirmação das relações entre as idéias delirantes e os atos violentos cometidos foi obtida pela entrevista direta, por meio dos registros médico-legais, e diretamente com a equipe de funcionários do serviço de saúde mental.

O grupo estudado foi selecionado da Casa de Custódia do município de Franco da Rocha, estado de São Paulo. Este estabelecimento recebe indivíduos que cometem crimes graves e são considerados inimputáveis em decorrência de transtorno psiquiátrico ${ }^{25}$.

Os pacientes do grupo comparado foram selecionados das enfermarias psiquiátricas de dois hospitais universitários (Unicamp e PUC-Campinas) e do hospital psiquiátrico Américo Bairral do município de Itapira, estado de São Paulo. Os sujeitos foram selecionados com base em seu diagnóstico de transtorno do espectro da esquizofrenia, mantendo atividade delirante e com base em dados obtidos diretamente na entrevista (conduzida por EHT) e nos antecedentes familiares, confirmando que eles não apresentavam história de crime violento. Todas estas informações foram confirmadas com a equipe de saúde mental e por meio de seus prontuários médicos.

Crime violento foi definido como crime que conduzisse à morte ou ao dano grave à vítima, como assassinato ou estupro, ou como ocorrências repetidas de grave ameaça de violência, como assaltos recorrentes.
Como deveria ser excluído do estudo casos de psicose induzida por substâncias psicoativas ou de transtorno psicótico breve, somente foram considerados os pacientes que tinham sido diagnosticados com transtorno do espectro da esquizofrenia há pelo menos cinco anos. A fim de evitar pacientes crônicos já com prejuízo cognitivo, deveriam, então, ter tempo de doença menor que 20 anos. Todos os sujeitos eram masculinos, sendo este o gênero predominante na instituição em que o grupo estudado foi selecionado.

Nenhum paciente foi considerado para este estudo quando havia possível história de lesão cerebral ou de retardo mental. Dez pacientes se recusaram a participar do grupo estudado e as razões dadas para essa recusa foram, principalmente, a falta de interesse, a ausência de algum "ganho" com a entrevista e o receio de que suas respostas poderiam interferir no resultado de laudo pericial para avaliação de cessação de periculosidade. Além disso, algumas entrevistas foram interrompidas porque os pacientes estavam comprometidos de maneira tão grave que a entrevista se tornara impraticável, principalmente em decorrência de prejuízo cognitivo ou pensamento incoerente.

A seleção dos instrumentos usados neste estudo foi fundamentada em revisão da literatura, tendo como base que os pacientes eram delirantes e, para o grupo estudado, além de delirantes tinham também história de crime violento. Conseqüentemente, os instrumentos foram selecionados, sempre que possível, quando tinham sido submetidos a estudos de validação e de confiabilidade. Foram utilizadas as escalas MMDAS $20,21,26$, MINI $^{27}$ (entrevista neuropsiquiátrica miniinternacional) e a PANSS (escala das síndromes positiva e negativa). Embora o MMDAS não tenha sido validado inteiramente no Brasil, após a tradução para o português realizada pelos autores deste artigo, a retradução para o inglês foi conduzida por um psiquiatra americano formado nos Estados Unidos e com grande experiência em psiquiatria clínica no Brasil (Dr. Sílvio Saidemberg). Além disso, durante as entrevistas executadas pelo primeiro autor (EHT), atenção especial foi dada em relação à compreensão das perguntas e dos termos pelos pacientes. Geralmente, a MMDAS foi bem aceita e compreendida pelos pacientes avaliados nesse estudo.

Para avaliar o grau de psicopatia, foram feitas perguntas da escala MINI, do capítulo de transtorno de personalidade anti-social, como: "Costumava ter comportamentos que os outros achavam irresponsáveis, como não pagar dívidas, agir impulsivamente ou não querer trabalhar para se sustentar? Freqüentemente fez coisas ilegais (mesmo que não tenha sido preso), como destruir propriedade alheia, roubar, vender drogas ou cometer um crime? Freqüentemente foi violento fisicamente, inclusive com sua companheira ou filhos? Freqüentemente mentiu, "passou a perna" ou enganou outras pessoas para obter dinheiro ou prazer ou men- 
tiu apenas para se divertir? Expôs pessoas a perigos sem se preocupar com elas? Não sentiu nenhuma culpa depois de ter mentido, ferido, maltratado, roubado alguém ou destruído propriedade alheia?". As respostas de ambos os grupos foram confirmadas com os dados contidos nos registros clínicos e médico-legais.

As variáveis primárias e secundárias do estudo foram: idade, gênero, escolaridade, estado civil, tempo de doença, diagnóstico clínico, comorbidade, perfil de sintomas clínicos, tipo de conteúdo de delírio e dimensões do delírio.

Para a realização do trabalho estatístico foi criado um banco de dados utilizando o programa estatístico SPSS, versão 7.5 e os procedimentos estatísticos envolveram análise descritiva, por meio de tabelas de freqüência, médias, medianas, desvios-padrão e intervalos e foram utilizados o teste qui-quadrado, o teste exato de Fisher e, em relação à comparação das escalas de avaliação de delírio (MMDAS) entre os grupos, foi utilizado o teste de Mann-Whitney. O nível de significância adotado foi de 5\%. Foi realizado modelagem final por meio de modelo multivariado.

Os sujeitos foram selecionados para o estudo somente após dar seu consentimento assinado. Todos receberam cópia do formulário de consentimento informado, que foi lido pelo pesquisador ou pelo próprio paciente, além de ter a oportunidade de fazer todas as perguntas antes da assinatura. Todos os pacientes investigados assinaram o termo de consentimento livre e esclarecido, foram orientados sobre a natureza do estudo e sobre a total garantia de sigilo. O estudo foi aprovado pelo Comitê de Ética em Pesquisa do Departamento de Psicologia Médica e Psiquiatria da Faculdade de Ciências Médicas da Universidade Estadual de Campinas (FCM-UNICAMP).

\section{RESULTADOS}

A população total da Casa de Custódia de Franco da Rocha no início desse estudo, com exceção da unidade de desinternação progressiva, consistia de 498 pacientes, sendo 91 mulheres (18\%) e 407 homens (82\%), com maior representação dos transtornos psicóticos, como a esquizofrenia, os transtornos delirantes, os transtornos psicóticos agudos e os transtornos esquizoafetivos $(n=288,57,8 \%)^{25}$.

A idade média do grupo estudado era de 38,0 \pm 9,5 anos e de 38,9 \pm 9,5 anos para o grupo comparado. A maioria dos pacientes em ambos os grupos era de solteiros (grupo estudado $=90 \%$; grupo comparado $=93,3 \%$ ) e de brancos (grupo estudado $=60 \%$; grupo comparado $=$ $73,3 \%)$. A duração média da doença no grupo estudado era de 15,8 \pm 7,5 anos, e de 16,5 \pm 9,1 anos no grupo comparado. Para o grupo estudado, o intervalo de tempo entre a avaliação do paciente na pesquisa e a ocorrência do crime foi de $6,3 \pm 4,3$ anos.
De acordo com os critérios diagnósticos da CID-10 e do DSM-IV, 21 (70\%) pacientes do grupo estudado foram diagnosticados como esquizofrenia paranóide, quatro (13,3\%) pacientes foram diagnosticados como esquizofrenia indiferenciada e cinco $(16,7 \%)$ pacientes foram diagnosticados como transtorno delirante. No grupo comparado, 29 $(96,7 \%)$ pacientes foram diagnosticados como esquizofrenia paranóide e um (3,3\%) paciente foi diagnosticado como esquizofrenia indiferenciada.

Os dois grupos foram comparados utilizando-se o MINI, para avaliar abuso e dependência de álcool e drogas na época da internação (grupo comparado) e na época do delito (grupo estudado), e nenhuma diferença estatística significativa foi encontrada entre os dois grupos (Tabela 1).

Tabela 1. Álcool e abuso de drogas para o dois grupos.

\begin{tabular}{|c|c|c|c|c|c|c|}
\hline & \multicolumn{2}{|c|}{$\begin{array}{l}\text { Grupo estudado } \\
(n=30) \\
\text { (no momento do } \\
\text { ato violento) }\end{array}$} & \multicolumn{2}{|c|}{$\begin{array}{c}\text { Grupo comparado } \\
\qquad(n=30) \\
\text { (no momento } \\
\text { da internação) }\end{array}$} & \multirow[t]{2}{*}{ Missing } & \multirow[t]{2}{*}{ p valor } \\
\hline & Sim & Não & Sim & Não & & \\
\hline Álcool & $7(53,8)$ & $6(46,1)$ & $7(43,7)$ & $9(56,2)$ & 31 & $0,5884^{*}$ \\
\hline Maconha & $7(70,0)$ & $3(30,0)$ & $3(25,0)$ & $9(75,0)$ & 38 & $0,0836^{* *}$ \\
\hline Cocaína & $1(33,3)$ & $2(66,7)$ & $2(50,0)$ & $2(50,0)$ & 53 & SS \\
\hline
\end{tabular}

* qui-quadrado; ** teste de Fischer; ss = sem significância.

Além disso, nenhuma diferença estatística significante foi encontrada no que diz respeito ao comportamento suicida, auto-agressivo ou anti-social.

Segundo a PANSS, ambos os grupos tiveram pontuação muito similares, conforme os resultados apresentados na Tabela 2.

Tabela 2. Comparação dos sintomas psicóticos/psiquiátricos entre os dois grupos.

\begin{tabular}{lccc}
\hline & $\begin{array}{c}\text { Grupo estudado } \\
(\mathbf{n}=30)\end{array}$ & $\begin{array}{c}\text { Grupo comparado } \\
(\mathbf{n}=30)\end{array}$ & pvalor $^{*}$ \\
\hline PANSS-G & $35,7 \pm 9,4(21,0-62,0)$ & $34,3 \pm 8,6(20,0-55,0)$ & 0,5860 \\
PANSS-P & $18,7 \pm 6,5(8,0-33,0)$ & $18,5 \pm 6,3(7,0-32,0)$ & 0,8769 \\
PANSS-N & $17,3 \pm 6,6(9,0-35,0)$ & $15,8 \pm 5,2(10,0-30,0)$ & 0,5505 \\
\hline
\end{tabular}

* p valor com base no teste de Mann-Whitney; PANSS- $G$ (psicopatologia global) = perda de interesse, ansiedade, tensão, hábitos e postura, depressão, falta da cooperaçăo etc.; PANSS-P (sintomas positivos) = delírio, desorganizaçăo conceitual, comportamento alucinatório, excitaçăo, grandiosidade, desconfiança etc.; PANSS - N (sintomas negativos) = afeto embotado, contato pobre, passividade, apatia, retraimento social etc.

De acordo com a MMDAS, o delírio persecutório foi o tipo de delírio mais comum presente em ambos os grupos. No grupo estudado, 21 (70\%) pacientes tiveram este tipo de delírio, enquanto nove $(30,0 \%)$ pacientes não tiveram. No grupo comparado, $23(76,7 \%)$ pacientes tiveram este tipo de delírio e sete $(23,3 \%)$ pacientes não tiveram. O segundo delírio mais comum encontrado foi do tipo "controle e influência", seguido pelo delírio de grandeza. Não houve nenhuma diferença estatística significativa entre os grupos em relação ao conteúdo. Mesmo quando somente os três 
tipos principais de delírios foram considerados (persecutório, controle e influência e de grandeza), não houve ainda nenhuma diferença estatística significativa entre os dois grupos (Tabela 3).

Tabela 3. Tipo de delírio de acordo com o conteúdo nos grupos estudado e comparativo.

\begin{tabular}{lccccc}
\hline \multirow{2}{*}{ Tipo de delírio } & \multicolumn{2}{c}{ Estudado } & & \multicolumn{2}{c}{ Comparado } \\
\cline { 2 - 3 } \cline { 5 - 6 } & $\mathbf{n}$ & $(\%)$ & & $\mathbf{n}$ & $(\%)$ \\
\hline Persecutório & 21 & $(72,4)$ & & 23 & $(76,7)$ \\
Grandeza & 3 & $(10,3)$ & & 4 & $(13,3)$ \\
Controle/Influência & 5 & $(17,3)$ & & 3 & $(10,0)$ \\
Total & $\mathbf{2 9}$ & $\mathbf{( 1 0 0 , 0 )}$ & & $\mathbf{3 0}$ & $\mathbf{( 1 0 0 , 0 )}$ \\
\hline
\end{tabular}

Das seis dimensões do delírio estudadas, foram encontradas diferenças estatísticas significativas entre os dois grupos para as três dimensões: "afeto negativo", "atuação no delírio" e "inibição de ação por causa do delírio". No caso "do afeto negativo", os pacientes do grupo estudado obtiveram pontuação de 1,1 \pm 1,2 (média \pm DP), enquanto os pacientes do grupo comparado obtiveram média de 2,5 $\pm 3,9$ pontos ( $p=0,0124)$. Em relação à "atuação no delírio", os pacientes do grupo estudado obtiveram média de 4,6 $\pm 0,9$ pontos, enquanto os pacientes do grupo comparado obtiveram média de 1,7 $\pm 1,3$ pontos ( $p=0,0001$ ). No que diz respeito à "inibição de ação por causa do delírio", a média obtida foi de 1,9 \pm 1,6 pontos para o grupo estudado e 3,3 $\pm 2,1$ pontos para o grupo comparado $(p=0,0076)$ (Tabela 4).

Tabela 4. Comparação das seis dimensões entre os grupos utilizando a MMDAS.

\begin{tabular}{lccc}
\hline MMDAS & $\begin{array}{c}\text { Grupo estudado } \\
(\mathbf{n}=29)\end{array}$ & $\begin{array}{c}\text { Grupo comparado } \\
(\mathbf{n}=\mathbf{3 0})\end{array}$ & p valor (*) \\
\hline Convicção & $5,9 \pm 1,6(2,0-8,0)$ & $5,3 \pm 1,7(2,0-8,0)$ & 0,2024 \\
Afeto negativo & $1,1 \pm 1,2(0,0-4,0)$ & $2,5 \pm 3,9(0,0-4,0)$ & 0,0048 \\
Atuação no delírio & $4,6 \pm 0,9(1,0-5,0)$ & $1,7 \pm 1,3(0,0-5,0)$ & $<0,0001$ \\
$\begin{array}{l}\text { Inibição de ação por } \\
\text { causa do delírio }\end{array}$ & $1,9 \pm 1,6(0,0-8,0)$ & $3,3 \pm 2,0(0,0-8,0)$ & 0,0069 \\
Preocupação & $2,4 \pm 0,9(1,0-4,0)$ & $2,5 \pm 0,9(1,0-4,0)$ & 0,6191 \\
Penetração & $1,9 \pm 0,7(1,0-3,0)$ & $2,2 \pm 0,5(1,0-3,0)$ & 0,2598 \\
\hline
\end{tabular}

$\left({ }^{*}\right)$ p valor com base no teste de Mann-Whitney.

Conforme a análise de regressão logística univariada, das seis dimensões estudadas, três mostraram diferença estatística significativa entre os grupos: "afeto negativo", "atuação no delírio" e "inibição de ação por causa do delírio" (Tabela 5). Utilizando regressão logística multivariada para as seis dimensões, considerando a presença ou não de ato violento, diferença estatística significativa foi encontrada na avaliação stepwise da dimensão "inibição de ação por causa do delírio". Para cada ponto adicional nesta dimensão, a chance de pertencer ao grupo estudado era $31 \%$ menor.
Tabela 5. Regressão logística univariada das seis dimensões da MMDAS.

\begin{tabular}{lccccc}
\hline Variável & $\begin{array}{c}\text { Grupo estudado } \\
(\mathbf{n})\end{array}$ & $\begin{array}{c}\text { Grupo comparado } \\
(\mathbf{n})\end{array}$ & p valor & OR & $95 \% \mathrm{Cl}$ \\
\hline Convicção & 29 & 30 & 0,2202 & 1,224 & $(0,886-1,690)$ \\
Afeto negativo & 29 & 30 & 0,0124 & 0,502 & $(0,292-0,861)$ \\
$\begin{array}{l}\text { Atuação } \\
\text { no delírio }\end{array}$ & 29 & 30 & $<0,0001$ & 4,091 & $(2,220-7,539)$ \\
$\begin{array}{l}\text { Inibição de } \\
\text { ação por causa }\end{array}$ & 29 & 30 & 0,0076 & 0,644 & $(0,466-0,889)$ \\
do delírio & & & & & \\
$\begin{array}{l}\text { Preocupação } \\
\text { Penetração }\end{array}$ & 29 & 30 & 0,5322 & 0,841 & $(0,488-1,449)$ \\
\hline
\end{tabular}

\section{DISCUSSÃO}

Ao utilizar uma amostra de casos graves e selecionados por conveniência (pacientes internados e sob medida de segurança), fez-se não representativo do universo da população geral de pacientes delirantes. No entanto, considerando os dados sociodemográficos e clínicos, as duas amostras investigadas no estudo foram muito semelhantes. Conseqüentemente, os grupos são comparáveis em relação à hipótese deste estudo, isto é, avaliar quais fatores estão associados com estar delirante e cometer crime violento.

$\mathrm{Na}$ avaliação da comorbidade de substância psicoativa, álcool e abuso de drogas e indicadores de psicopatia, é possível que alguns pacientes tentassem esconder ou negar os sintomas, pois poderiam acreditar que a entrevista acabaria por afetar indiretamente sua condição, no que se refere à avaliação de periculosidade. Sabendo da importância destas comorbidades para a internação ou para a ocorrência de um crime, esta condição foi cuidadosamente estudada. Nesse sentido, a respeito do uso e do abuso de substância, revisão cuidadosa dos registros clínicos e legais foi executada. Tais dificuldades da avaliação foram reconhecidas como limitações freqüentes nos estudos similares realizados neste tipo de população psiquiátrica ${ }^{6,7,15}$.

No presente estudo, a hipótese de alguns autores que consideram possível efeito do álcool nos crimes violentos por pacientes psicóticos (e pela atividade delirante) não foi confirmada ${ }^{11,15}$. Certamente, o presente estudo tende a sugerir associação entre a psicopatologia do delírio e o ato violento, independentemente do uso do álcool ou da droga. Entretanto, possível viés deve ser considerado: alguns pacientes poderiam ter tentado esconder ou negado tal relação com substâncias ou comportamentos anti-sociais (Tabela 1).

Considerando o perfil de diagnóstico clínico encontrado em ambos os grupos, o fato de ter cinco indivíduos com diagnóstico de transtorno delirante no grupo estudado e nenhum no grupo comparado pode sugerir alguma heterogeneidade diagnóstica entre os dois grupos. No entanto, o número absoluto de sujeitos com diagnóstico de trans- 
torno delirante foi razoavelmente baixo. Isso pode sugerir que no grupo estudado, como indivíduos com transtornos delirantes são menos comprometidos, em termos de estrutura geral da personalidade, podem ter, então, propensão maior para cometer ato violento. Entretanto, nenhuma diferença foi encontrada entre os dois grupos, no que diz respeito à psicopatologia geral avaliada pela PANSS. De fato, foram muito semelhantes e o grupo estudado teve pontuação maior na escala de sintomas negativos, embora esta diferença não fosse estatisticamente significante. Conseqüentemente, é improvável que a presença de pacientes com transtornos delirantes no grupo estudado represente alguma diferença significativa entre os grupos.

No que diz respeito aos pensamentos suicidas no passado (pensar em se matar em alguma época da vida), alta freqüência desta circunstância foi encontrada em ambos os grupos (56,7\% no grupo estudado e 73,3\% no grupo comparado), e não houve nenhuma diferença estatística significante. Este fato pode estar relacionado à própria circunstância imposta pela gravidade da enfermidade mental.

A avaliação do estado mental destes pacientes, de acordo com a PANSS, mostrou, como mencionado anteriormente, que os grupos eram razoavelmente homogêneos. Na avaliação de sintomas positivos e negativos, os pacientes de ambos os grupos foram pontuados como completamente sintomáticos ${ }^{28}$.

$\mathrm{Na}$ avaliação realizada utilizando a MMDAS, em relação aos aspectos do delírio, o tipo persecutório foi o mais freqüente em ambos os grupos (70\% no grupo estudado e $76,7 \%$ no grupo comparado). Este perfil foi encontrado em vários estudos em que transtornos delirantes e violência foram pesquisados ${ }^{4,21}$. No grupo estudado, o segundo tipo mais freqüente de delírio foi de "controle ou influência", seguido pelo delírio de grandeza. No grupo comparado, o segundo tipo mais comum foi de grandeza seguido pelo de "controle ou influência". Não houve nenhuma diferença estatística significativa entre os dois grupos.

A respeito das dimensões do delírio avaliadas nesta escala (convicção, atuação no delírio, afeto negativo, inibição de ação por causa do delírio, penetração e preocupação), os resultados foram semelhantes aos encontrados em estudos anteriores que avaliaram pacientes delirantes de categorias diagnósticas diferentes ${ }^{20}$.

Das seis dimensões avaliadas, a diferença em relação à "atuação no delírio" era esperada, já que esta era a condição para pertencer ao grupo estudado. Em relação à "inibição de ação por causa do delírio" (por exemplo: deixar de assistir televisão, de comer, de ir ao trabalho ou ao médico por causa do delírio), o grupo comparado obteve pontuação significativamente mais alta. Além disso, a avaliação estatística com regressão logística multivariada realizada com a MMDAS, considerando a presença ou a ausência de um ato violento, obteve valor estatístico significante na avaliação de stepwise para a dimensão "inibição de ação por causa do delírio", isto é, para cada ponto adicional nesta dimensão, a probabilidade de o indivíduo pertencer ao grupo estudado diminui 31\%. Este resultado sugere que nos pacientes delirantes, cujos contextos do delírio inibem suas ações, pode ocorrer tendência de efeito protetor de ações potencialmente prejudiciais associadas com o próprio delírio.

"O afeto negativo", definido como estar se sentindo infeliz, amedrontado, ansioso ou irritado em decorrência do delírio, teve, de modo igual, pontuação mais elevada no grupo comparado. Este resultado questiona a impressão geral comum no meio médico, psicológico e psiquiátrico, de que o paciente delirante sentindo-se amedrontado, ansioso ou aterrorizado é mais provável de cometer crime violento. Curiosamente, os resultados deste estudo sugerem o contrário.

É igualmente possível que "o afeto negativo" possa ser indicativo de depressão e de baixa auto-estima, que, de certa forma, está relacionada a atos menos violentos. Uma outra possibilidade é de que o paciente delirante que comete crime violento esteja envolvido tão intensamente em seu delírio, que os afetos negativos seriam vivenciados em menor intensidade.

\section{CONCLUSÃO}

A dimensão "afeto negativo" pareceu ser relevante e mostrou que os pacientes do grupo estudado tenderam a ser menos amedrontados, ansiosos, infelizes ou irritados em conseqüência do delírio. A dimensão "inibição de ação por causa do delírio" tem valor claramente significativo. É possível que os delírios que conduzam a maior "inibição de ações" no sujeito em seu mundo, de certa maneira, de modo igual, inibam ações agressivas e potencialmente violentas resultantes do delírio.

No geral, o presente estudo não confirmou os resultados relatados na literatura que tratou do tópico delírio e crime violento relacionados à comorbidade do uso de álcool e droga e do tipo de delírio, e adiciona novos aspectos no que diz respeito ao delírio e suas dimensões, que, embora modesto, parecem ser relevantes.

A continuação desta linha de pesquisa, com o objetivo de identificar os aspectos da sintomatologia que possam de certa maneira estar relacionados ao comportamento potencialmente violento, pode contribuir para a compreensão deste fenômeno e colaborar no desenvolvimento de possíveis ações preventivas.

\section{AGRADECIMENTOS}

Agradece-se a especial atenção do Dr. Sílvio Saidemberg, médico psiquiatra, ex-docente da FCM da Puc-Campinas, que muito colaborou na fase de retradução e adaptação cultural da escala MMDAS. 


\section{REFERÊNCIAS}

1. Arboleda-Florez J. Contemporary forensic psychiatry: a review. Advanc Psychiatry. 2005:2:173-9.

2. Binder RL. Are the mentally ill dangerous? J Am Acad Psychiatry Law. 1999;27:189-201.

3. Mullen PE. Forensic mental health. Br J Psychiatry. 2000;176:307-11.

4. Walsh E, Buchanan A, Fahy T. Violence and schizophrenia: examining the evidence. Br J Psychiatry. 2001;180:490-5.

5. Monahan J. Mental disorder and violent behavior: perceptions and evidence. Am Psychol. 1992;47:511-21.

6. Arboleda-Florez J. Mental illness and violence: an epidemiological appraisal of the evidence. Can J Psychiatry. 1998;43(10):986-96.

7. Marzuk PM. Violence, crime, and mental illness. How strong a link? Arch Gen Psychiatry. 1996;53:481-6.

8. Chaves AC, Shirakawa I. Escala das síndromes positiva e negativa e seu uso no Brasil. Rev Psiq Clin. 1998;60:337-43.

9. Eronen M, Hakola P, Tiihonen J. Mental disorders and homicidal behavior in Finland. Arch Gen Psychiatry. 1996;53:497-501.

10. Hogan MF. The president's new freedom commission: recommendations to transform mental health care in America. Psychiatr Serv. 2003;54:1467-74.

11. Shaw J, Hunt IM, Flynn S, Meehan J, Robison J, Bickley H, et al. Rates of mental disorder in people convicted of homicide. National clinical survey. Br J Psychiatry. 2006;188:143-7.

12. Taylor PJ, Gunn J. Violence and psychosis. I. Risk of violence among psychotic men. Br Med J. 1984;288:1945-9.

13. Taylor PJ, Gunn J. Homicides by people with mental illness: myth and reality. Br J Psychiatry. 1999:174:9-14.

14. Teplin LA. The prevalence of severe mental disorder among urban jail detainees: comparison with the epidemiologic catchment area program. Am J Public Health. 1990;80:663-9.

15. Vevera J, Hubbard A, Vesely A, Papezova H. Violent behavior in schizophrenia. Retrospective study in four independent samples from Prague, 1949 to 2000. Br J Psychiatry. 2005;187:426-30.
16. Wallace C, Mullen P, Burgess P, Palmer S, Ruschena D, Browne C. Serious criminal offending and mental disorder. Case linkage study. Br J Psychiatry. 1998;172:477-84.

17. Stuart HL, Arboleda-Florez JE. A public health perspective on violence offenses among persons with mental illness. Psychiatr Serv. 2001;52:654-9.

18. Taylor PJ, Leese M, Williams D, Butwell M, Daly R, Larkin E. Mental disorder and violence. A special (high security) hospital study. Br J Psychiatry. 1998;172:218-26.

19. Link BG, Stueve A. Psychotic symptoms and the violent/illegal behavior of mental patients compared to community. In: Monahan J, Steadman H, editors. Violence and mental disorder: developments in risk assessment. Chicago, IL: University of Chicago Press; 1994. p. 137-59.

20. Cheung P, Schweitzer I, Crowley K, Tuckwell V. Violence in schizophrenia: role of hallucinations and delusions. Schizophr Res. 1997;26(2-3):181-90.

21. Appelbaum PS, Robbins PC, Roth LH. Dimensional approach to delusion: comparison across types and diagnoses. Am J Psychiatry. 1999;156(12):1938-43.

22. Appelbaum PS, Robbins PC, Monahan J. Violence and delusions: data from the Macarthur Violence Risk Assessment Study. Am J Psychiatry. 2000;157(4):566-72.

23. Oliveira LM. Período médico-legal da esquizofrenia. In: Serafim AP, Barros DM, Rigonatti SP, et al., editors. Temas em psiquiatria forense e psicologia jurídica. São Paulo: Vetor Editora, 2006; p. 33-8.

24. Stompe T, Ortwein-Swoboda G, Schanda H. Schizophrenia, delusional symptoms, and violence: the threat/control-override concept reexamined. Schizophr Bull. 2004;30(1):31-44.

25. Teasdale B, Silver E, Monahan J. Gender, threat/control-override delusions and violence. Law Hum Behav. 2006;30:649-58.

26. Teixeira EH, Dalgalarrondo P. Crime, diagnóstico psiquiátrico e perfil da vítima: um estudo com uma amostra de uma Casa de Custódia de São Paulo. J Bras Psiquiatr. 2006;55(3):190-4.

27. Buchanan A, Reed A, Wessely S, Garety P, Taylor P, Grubin D, et al. Acting on delusions. II: the phenomenological correlates of acting on delusions. Br J Psychiatry. 1993;163:77-81.

28. Amorin P, Lecrubier $Y$, Weiller E, Hergueta T, Sheehan D. Psychotic disorders: procedural validity of the Mini International Neuropsychiatric Interview. Eur Psychiatry. 1998;13:26-34.

29. Steinert T, Wolfle M, Gebhardt RP. Measurement of violence during in-patient treatment and association with psychopathology. Acta Psychiatr Scand. 2000;102:107-12. 